\title{
Biomechanics of a Pedestrian Accident Reconstruction*
}

\author{
Ricardo J.F. Portal, J. Pereira Dias, Luis G. Sousa
}

\begin{abstract}
Pedestrians represent about $20 \%$ of the overall fatalities in Europe's road traffic accidents, as well as in Portugal. An in-depth investigation, accident reconstruction, is mandatory in order to analyze with care fundamental aspects of an accident. Detailed information about the injuries and injury causation mechanisms are with great use for it. Accident reconstruction computational techniques using pedestrian biomechanical models are used to evaluate the accident conditions that lead to major pedestrian injuries, such as the vehicle's speed and the impact location. For biomechanical injury criterions, the AIS (Abbreviated Injury Scale), the HIC (Head Injury Criterion) and some other injury criterions are used.
\end{abstract}

Pedestrian's height, weight, gender, age, vehicle geometry (or category) and travelling speed, significantly influence the pedestrian injuries in the event of a road collision.

The use of injury scales and biomechanical criterions in the in-depth accident investigation of road accidents, such as AIS, can improve significantly the quality of the reconstruction process.

The computer simulation of vehicle-to-pedestrian collisions is fundamental for the study of the dynamics of these road accidents. Where did the pedestrian is when the vehicle hits him? Normally, there is no simple and clear answer. The pedestrian, if survives, states that he uses the crossway, the driver of the vehicle states otherwise, that the location of the pedestrian was much later than the pedestrian crossing.

This work presents an accident where only the use of accident reconstruction specialized software, PC-Crash, conjugated with all available information allowed the determination of the vehicle's speed and the Location of the pedestrian.

\section{INTRODUCTION}

Every year about 1.2 million people die and more than 50 million are injured in road accidents. In Europe (Fig. 1), 25300 people lost their lives in 2017, which is $20 \%$ fewer than in 2010, more than $66 \%$ fewer than in 1991 (data collected from [1-2]). While this trend represents a great

Ricardo J.F. Portal are with GIMOSM - Grupo de Investigação em Modelação e Optimização de Sistemas Multifuncionais, ISEL - Instituto Superior de Engenharia de Lisboa, Av. Conselheiro Emídio Navarro 1, 1959-007 Lisboa, Portugal. (00-351-218-317-000; fax: 00-351- 218-317213; e-mail: rportal@dem.isel.ipl.pt).

Ricardo J.F. Portal is with icollision - Tecnologias e Consultoria para a Análise Científica de Acidentes, Av. Elias Garcia, $1415^{\circ}$ Dto, 1050-099, Lisboa, Portugal. (00-351-925 895 409; email: ricardo.portal@icollision.pt).

J. Pereira Dias and Luis G. Sousa are with IST - Instituto Superior Técnico, Universidade de Lisboa, Av. Rovisco Pais, 1049-001 Lisboa, Portugal.

Ricardo J.F. Portal, J. Pereira Dias and Luis G. Sousa are with LAETA, IDMEC - IST Instituto Superior Técnico, Universidade de Lisboa, Av. Rovisco Pais 1, 1049-001 Lisboa, Portugal. evolution, the EU objective of halving road fatalities between 2010 and 2020 will be very challenging.

It is estimated that 135000 people were seriously injured in 2017, including a large proportion of vulnerable users: pedestrians $(21 \%)$, cyclists, motorcyclists and mopeds riders $(25 \%)$. Beside the victims, road fatalities and injuries also affect the society, with a significant socio-economic cost [2].

Figure 1. EU Road fatalities evolution, 1991-2017.

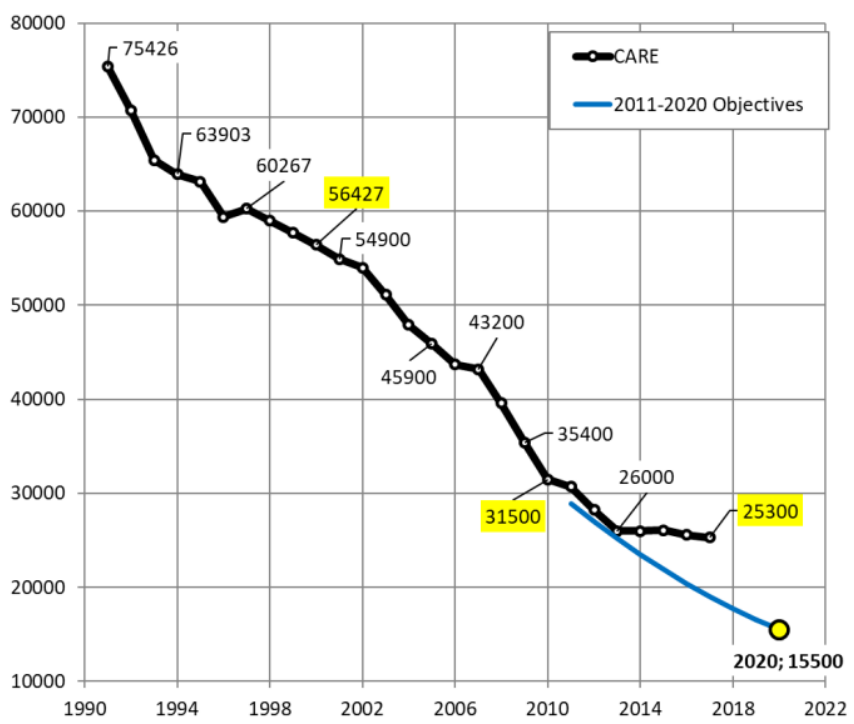

Since 2013 that there has been a stagnation of road fatalities, which is certainly related to the number of deaths of motorcyclists and pedestrians.

In order to better understand the causes of accidents and reduce the number of casualties, new security measures should be implemented, notably regarding the road design and the placement of light and vertical signaling. The correct behavior of drivers and pedestrians also has an important role to play. The accident reconstitution can effectively contribute to the determination of responsibilities and consequently to the development of new solutions for the structural components of vehicles and new pedestrian safety devices such as the optimization of the hood structure, the elevation of the hood and the pedestrian airbags.

A pedestrian accident is a progressive sequence of impacts between the pedestrian and the automobile. Most pedestrian collisions result from frontal collisions, as such the vehicle's front structures, such as the hood, the bumper or the windscreen, are responsible for most of the injuries checked. A first contact occurs, in which the leg of the pedestrian is hit by the bumper of the vehicle. The dynamic of the impact leads to the impact of the thigh and the pedestrian basin in the 
vehicle and the posterior impact of the upper regions of the body, such as the trunk and arms, and finally the head of the pedestrian with the hood and the windscreen (or the A-pillar) of the vehicle. Although the sequence described is the most usual, except for situations where the impact occurs with the lateral area of the vehicle, the injuries of the pedestrian have different degrees of gravity, related to the type of vehicle, the shape of the front of the vehicle, the impact velocity, as well as the biomechanical characteristics of the pedestrian, such as height, weight and age.

\section{ACCIDENT RECONSTRUCTION METHODS}

Accident reconstruction involving pedestrians can be complex and, in general, requires the use of $3 \mathrm{D}$ biomechanical models of the human body [3]. Multibody dynamics has been used in the development of these models successfully [3-6]. The development of vehicle and pedestrian models and its use in computational tools based in multibody [8] systems dynamics are essential.

Depending of the accident complexity, different methodologies have been used to analyze it, from the classical analytical equations like the ones resumed by Eubanks and Haight [9], to the use of detailed computational pedestrian models as those including in commercial software PC-Crash [10] or Madymo [11]. With these tools the impact speed can be determined from the projection distance with analytical tools or PC-Crash, but more complex tools should be used to determine speed from the injuries, what is especially important for fatal accidents. The influence of the vehicle geometry and stiffness characteristics is another important aspect, where the influence of the vehicle stiffness has been determined using a combined multibody-finite elements approach using Madymo. Finite Element Method is being gradually integrated in PC-Crash and in several other models (like THUMS) and software developments, like LSDyna [12].

The classic methods of analysis, for example the Searle's, Simms and Wood's or the University of Northwestern models, are simple mathematical models that can only be used under well-defined conditions, such as the position of the pedestrian relative to the vehicle, the Knowledge of the projection distance or the speed of the vehicle at the time of the impact. These last two variables are often unknown, and their calculation required at the same time. Thus, the role of the scientific reconstitution is fundamental for the correct characterization of the run-over, where the technical characteristics of the vehicle and the morphological of the pedestrian are considered, the local configuration of the accident, any braking and/or skid marks or traces resulting from the accident, such as glass and plastic parts of the vehicle that rans over or even blood and organic tissues of the pedestrian. It is also considered the rest positions of the vehicle and the of the pedestrian and he's medical records, since it is usual that the pedestrian injuries indicate the possible dynamic scenarios.

\section{REAL PEDESTRIAN ACCIDENT RECONSTRUCTION}

In the study of real pedestrian related accidents, where the conjugation of all the case information should be considered in the analysis, such as the characteristics of the road (coefficient of friction, inclination, curvature, physical elements, braking marks and/or skidding or traces resulting from the accident), the meteorological conditions, the technical characteristics of the vehicle and the morphological of the pedestrian, it is imperative to use computational tools specialized in the traffic road reconstruction. Gathering all available information becomes possible to simulate the scenarios indicated by eventual witnesses, determining the closest scenario to reality and refuting the scenarios devoid of physical meaning. The variables normally important for the judicial decision usually relates to the determination of the accident dynamics, the vehicle's crash speed and the location of the pedestrian on the road. If there are braking tracks or indications that there was braking, it can also be calculated the travelling speed prior to such braking.

In this section, a real pedestrian accident reconstruction performed with PC-Crash is presented.

The scenario created in PC-Crash to analyze the accident is depicted in Fig. 2, where the pedestrian position and the vehicle's rest position are indicated.

In this case, in contrast to usual, the testimonies are almost similar, a witness states that the vehicle driven by the pedestrian was the second on the left side, contrary to what the pedestrian himself claims. Allegedly, the worker is walking to his vehicle when a small vehicle runs over him.

There are no braking and/or skid marks and the position of the traces resulting from the accident aren't registered.

The impact in the pedestrian lets the vehicle with permanent deformations, clearly visible in Fig. 3, the amount of deformation, particularly the breaking of the windshield and the fact that he collides with the roof, makes the investigator suspect that the vehicle's speed is in the order of $80 \mathrm{~km} / \mathrm{h}$. The trajectory of the pedestrian is noticeably well defined.

Figure 2. 3D scenario for PC-Crash simulations

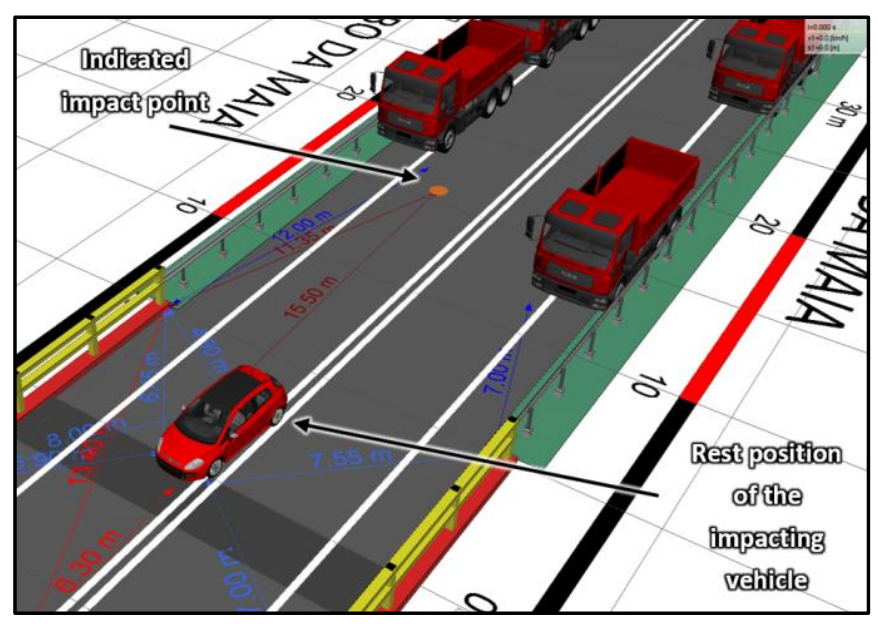

The 40-year old pedestrian, 1,83-meter-high, and a mass of 85 kilograms, was injured during the collision and probably in the road impact. Open cervical trauma; dislocation of the left shoulder; blunt thoracic trauma with bilateral pulmonary contusion; lesions of the left shoulder and in the glenoid cavity; brachial plexus injury reached the 
cubital, radial and circumflex nerve; a dislocation of a left leg muscle; and several abrasions on the body.

Figure 3. Vehicle damage produced by the impact with the pedestrian
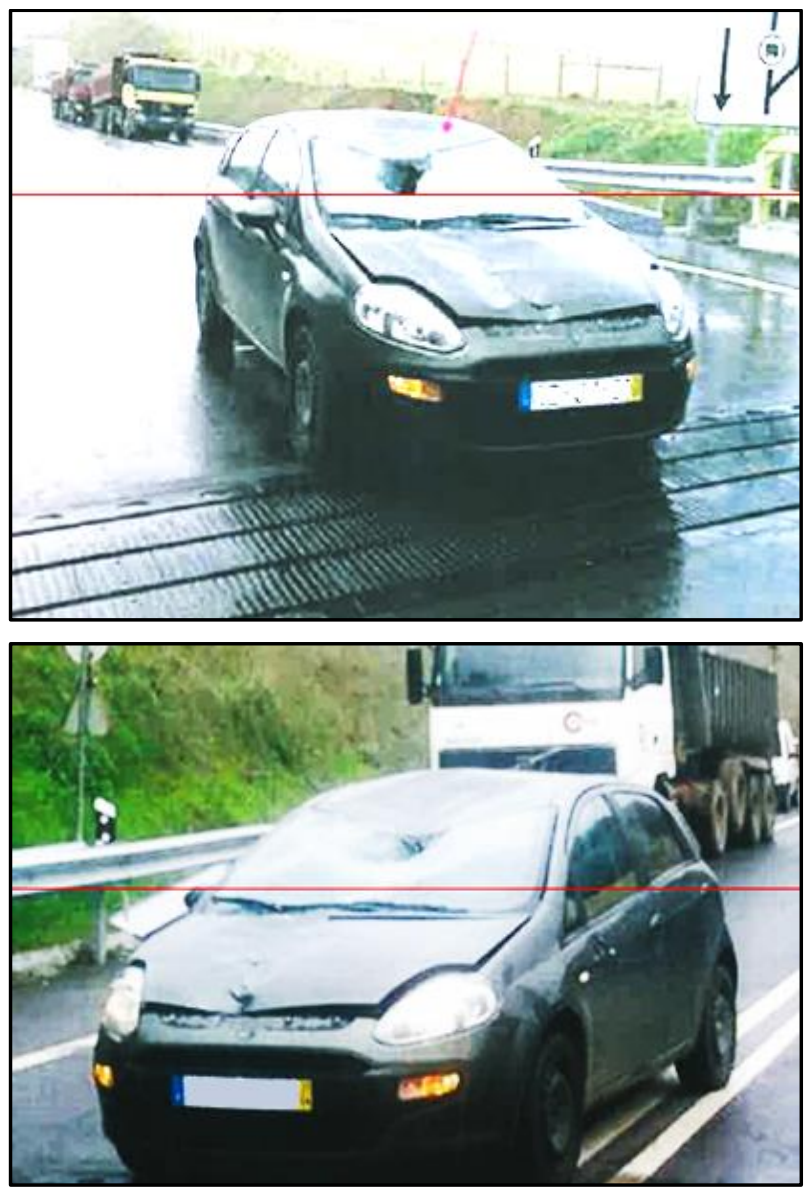

The fact that no injuries of the lower limbs are indicated and the fact that no significant deformations of the bumper of the vehicle is present, as it would be expected, may indicate that the pedestrian has noticed the vehicle before being hit and has attempted to avoid the collision: through a small leap or increasing his waking speed, being hit in the aerial phase of a gait.

In a first analysis, analytical methods were used to evaluate the vehicle speed, assuming a certain vehicle projection distance (approximately 15 meters) indicated by the witnesses. Even considering a vehicle speed of $50 \mathrm{~km} / \mathrm{h}$, and if the driver only sees the pedestrian when the vehicle hits him, the driver needs approximately 32 meters to completely stops the vehicle. This led to a sub-evaluation of the vehicle speed, and the indicated impact position is no possible. So, for the accident reconstruction using PC-Crash, the area of impact was retracted a few meters and the velocity range increased.

The vehicle and pedestrian models are presented in Fig. 4 and reflect both vehicle and pedestrian characteristics.

The computational simulation led to the determination of the point of impact, and the vehicle speed at the point of impact. It was considered a pedestrian speed at the point of impact of $2 \mathrm{~km} / \mathrm{h}$. the impact occurs with the vehicle traveling at $70 \mathrm{~km} / \mathrm{h}$, and the vehicle traveled 26 meters from the impact to its immobilization. The projection distance of the pedestrian was 32 meters.

Figure 4. Vehicle and pedestrian models: (left) pedestrian model; (center) visulization model; triangulated mesh model (right)

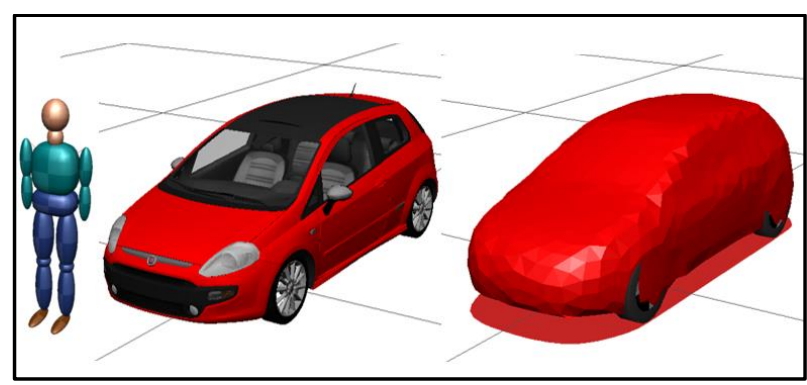

Fig. 5 depicts two stroboscopic perspective of the accident dynamics, from the point of impact to the immobilization of the vehicle and the pedestrian. It can be noticed that the initial point of impact significantly change, being anticipated about ten meters.

Figure 5. Estroboscopic perspectives of the determinated accident dynamics
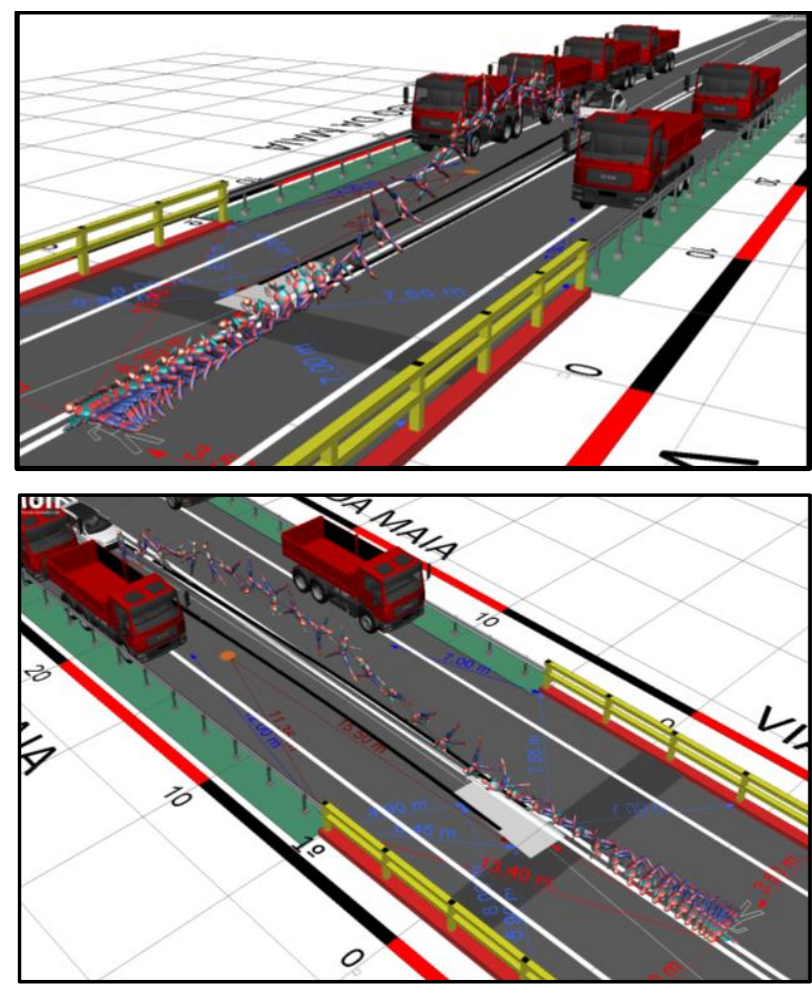

\section{CONCLUSION}

Pedestrian accident reconstruction can be performed with simplified models or with more complex ones, the choice that the reconstructionist must do relates with the accident complexity, the amount of information that he has and the predicted accident configuration.

Analytical methods prove to give accurate results for specific and simpler accident scenarios, mainly to determine impact speed and allow a fast way to determine a start value 
for the investigation. When the reconstructionist needs to solve more complex accidents, he must use different formulations that enables the correlation between skid marks, vehicle and pedestrian rest positions, energy dissipation and evidences gathered at accident scene. In these cases, becomes mandatory that experts in accident reconstruction use specialized software.

\section{REFERENCES}

[1] Road safety evolution in EU. CARE - Community database on Accidents on the Roads in Europe. Mars 2012.

[2] Road safety evolution in EU. CARE - Community database on Accidents on the Roads in Europe. April 2018.

[3] J. Yang, Crash Analysis - The Pedestrian Accident Reconstruction, Chalmers University of Technology, Göteborg, Sweden, 2002.

[4] M.T.Silva, J.C.Ambrósio, "Pedestrian Impact and Run Over Using a Multibody Simulation Tool", International Journal of Crashworthiness, Vol. 4(3), pp. 261-271, 1999.

[5] P.M.Lima, R.F.Portal, J.P. Dias, "Models for Pedestrian Accident Reconstruction". II International Conference On Computational Bioengineering. H. Rodrigues et al. (Eds.). Lisboa, Portugal, September 14-16, 2005.

[6] R.F.Portal, J.P.Dias. "Multibody Models For Vehicle Accident Reconstruction". ECCM 2006 - III European Conference on Computational Mechanics - Solids, Structures and Coupled Problems in Engineering. C.A. Mota Soares et al. (eds.). Lisbon, Portugal, June $5-8,2006$.

[7] J.P.Dias, R.F.Portal, A.C.Freitas, "Causas da sinistralidade pedonal em Portugal e medidas para a sua redução". V Congresso Rodoviário Português. Estrada 2008 - Sistemas Inteligentes, Logística e Turismo. Centro de Congressos do Estoril, Estoril, Portugal. March 12-14 2008. (in Portuguese)

[8] P.E.Nikravesh, Computer Aided Analysis of Mechanical Systems, Prentice-Hall, Englewood Cliffs, NJ, 1988.

[9] J.J.Eubanks, W.R.Haight, "Pedestrian Involved Traffic Collision Reconstruction Methodology”. Society of Automotive Engineer. Paper 921591, 1992.

[10] Datentechnik, S., PC-Crash, A Simulation program for Vehicle Accidents, Operating Manual, Version 12.0, Linz, Austria, 2018.

[11] TNO, Madymo v6.2 Theory Manual, Delft, Netherlands, June 2004

[12] C. Bastien, M. Orlowski, M. Bhagwani, "Validation of Thums Human Model Throw Distance in Pedestrian Accident Scenarios", 11th European LS-DYNA Conference, Salzburg, Austria, 2017. 